\title{
The Exponentiated Weibull-Geometric Distribution: Properties and Estimations
}

\author{
Younshik Chung ${ }^{1, a}$, Yongbeen Kang ${ }^{a}$ \\ ${ }^{a}$ Department of Statistics, Pusan National University, Korea
}

\begin{abstract}
In this paper, we introduce the exponentiated Weibull-geometric (EWG) distribution which generalizes twoparameter exponentiated Weibull (EW) distribution introduced by Mudholkar et al. (1995). This proposed distribution is obtained by compounding the exponentiated Weibull with geometric distribution. We derive its cumulative distribution function (CDF), hazard function and the density of the order statistics and calculate expressions for its moments and the moments of the order statistics. The hazard function of the EWG distribution can be decreasing, increasing or bathtub-shaped among others. Also, we give expressions for the Renyi and Shannon entropies. The maximum likelihood estimation is obtained by using EM-algorithm (Dempster et al., 1977; McLachlan and Krishnan, 1997). We can obtain the Bayesian estimation by using Gibbs sampler with Metropolis-Hastings algorithm. Also, we give application with real data set to show the flexibility of the EWG distribution. Finally, summary and discussion are mentioned.

Keywords: Bayesian estimation, EM Algorithm, exponentiated Weibull distribution, exponentiated Weibull geometric distribution, geometric distribution, Gibbs sampler, hazard function, MetropolisHastings algorithm, MLE, Markov chain Monte Carlo (MCMC).
\end{abstract}

\section{Introduction}

Several distributions have been proposed to model lifetime data. Mudholkar et al. (1995) have introduced a two-parameter exponentiated Weibull (EW) distribution as an extension of Weibull distributions. Other statistical properties of EW distributions have been examined by Nassar and Eissa (2003, 2004). They had derived Bayes estimates for the two shape parameters, reliability and failure rate functions of EW distributions. Kim et al. (2011) have investigated the Bayesian estimator for the EW model under progressive Type II censoring. Jung and Chung (2013) studied the Bayesian prediction of EW distribution based on progressive type II censoring.

The Weibull and EW distributions has been widely used in survival analyses and reliability studies due to their simplicity. However, they did not provide a reasonable parametric fit in some practical applications. Recently, attempts have been made to define new families of probability distributions that extend well-known families of distributions and that time provide great flexibility in modeling data in practice. Alexander et al. (2012) mentioned that new techniques for building meaningful distributions are widely investigated, including the two-piece approach by Hansen (1994), the pertubation approach of Azzalini and Capitanio (2003), the generator approach by Eugene et al. (2002) and compounding approach by Barreto-Souza et al. (2011). In general, compounding approach is as follows; let $Y$ be the lifetime of a system, defined by

$$
X=\min _{1 \leq i \leq Z}\left(Y_{i}\right) \quad \text { or } \quad X=\max _{1 \leq i \leq Z}\left(Y_{i}\right)
$$

\footnotetext{
${ }^{1}$ Corresponding author: Professor, Department of Statistics, Pusan National University, Jangjeon-Dong, Geumjeong-Gu, Pusan 609-735, Korea. E-mail: yschung@pusan.ac.kr
} 
where the distribution of $Y_{i}$ belongs to one of the well-known lifetime distributions and the random variable $Z$ has one of the discrete distributions. Then the unconditional marginal distribution of $X$ has been considered as a new family of lifetime distribution. Moreover, generalized Beta-type distributions based on the generator approach are studied by Cordeiro et al. (2011) and Bidram et al. (2011). For more details, see Nadarajah et al. (2013).

In this article, we propose a new three-parameter distribution, referred to as the exponentiated Weibull geometric (EWG) distribution based on the compounding approach, which contains as special sub-models Weibull (W), exponentiated Weibull (EW) and generalized exponential (GE) distribution. Here, we compound $X=\min _{1 \leq i \leq Z}\left(Y_{i}\right)$ with the geometric distribution $Z$ where $Y_{i}$ is distributed to the two-parameter exponentiated Weibull distributions. We showed that the hazard function of EWG distributions can be decreasing, increasing and bathtub-shaped. Several properties of EWG distributions such as quantiles, moments, maximum likelihood estimation (MLE) procedure via an EM algorithm, Bayesian estimation, Renyi and Shannon entropies and moments of order statistics are obtained. Also, two real lifetime data set are used for comparing the proposed EWG model with the competing models using AIC, BIC and graphs. Recently, Mahmoudi and Shiran (2012) proposed the similar exponentiated Weibull geometric using the same compounding approch based on the threeparameter exonentiated Weibull distributions but they used $X=\max _{1 \leq i \leq Z}\left(Y_{i}\right)$ which is different to our proposed techique $X=\min _{1 \leq i \leq Z}\left(Y_{i}\right)$. Also, Mahmoudi and Shiran (2012) studied the properties of its different types of submodels using MLE only without Bayesian estimates and real data to be explained for comparing models.

The paper is organized as follows. We propose the EWG distribution in Section 2. We obtain the cumulative distribution function (cdf), survivor function, hazard functions and the probability density function (pdf) of the order statistics, quantiles, moments of order statistics and Renyi and Shannon entropies in Section 3. The methods for obtaining MLE using EM algorithm and Bayesian estimators using MCMC method are explained in Section 4. We obtain the MLE and Bayesian estimates of parameters from the EWG model based on real lifetime data for application and compare the EWG model with the competing models using AIC, BIC and graphs in Section 5. Finally, summary and discussion are mentioned in Section 6.

\section{The EWG Distribution}

Suppose that $\left\{Y_{i}\right\}_{i=1}^{Z}$ are independent and identically distributed (iid) random variables from the Exponentiated Weibull(EW) distribution $\operatorname{EW}(\alpha, \beta)$ with $\alpha>0, \beta>0$ proposed by Mudholkar et al. (1995) and the probability density distribution (pdf) and the cumulative distribution(cdf) are given by

$$
g(\mathbf{y} \mid \alpha, \beta)=\alpha \beta y^{\alpha-1} e^{-y^{\alpha}}\left(1-e^{-y^{\alpha}}\right)^{\beta-1}, \quad y>0
$$

and

$$
G(\mathbf{y} \mid \alpha, \beta)=\left(1-e^{-y^{\alpha}}\right)^{\beta}, \quad y>0,
$$

respectively. And $Z$ is a discrete random variable having a geometric distribution with the probability function

$$
P(z \mid p)=(1-p) p^{z-1}, \quad z=1,2, \ldots, 0 \leq p<1 .
$$


$(0.5,0.8)$

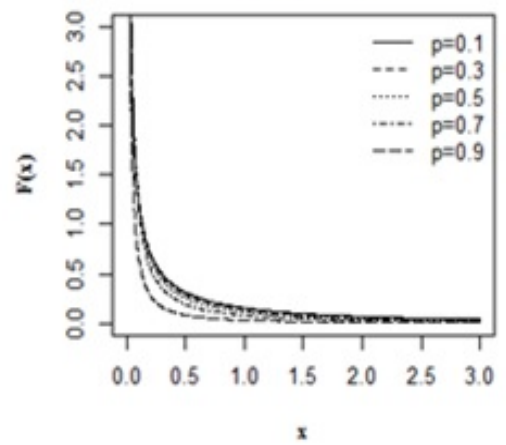

$(2,2)$

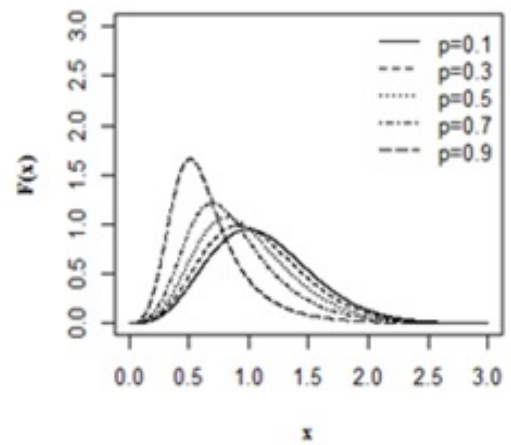

$(1.2,1.5)$

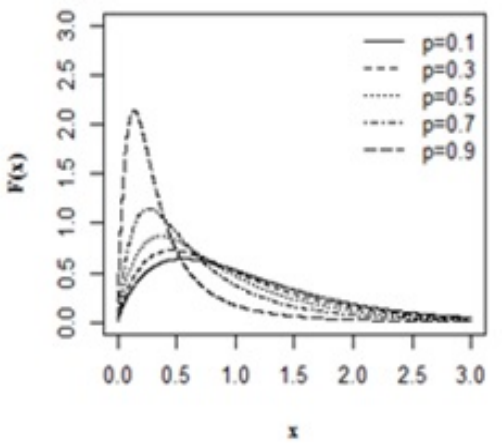

$(4,4)$

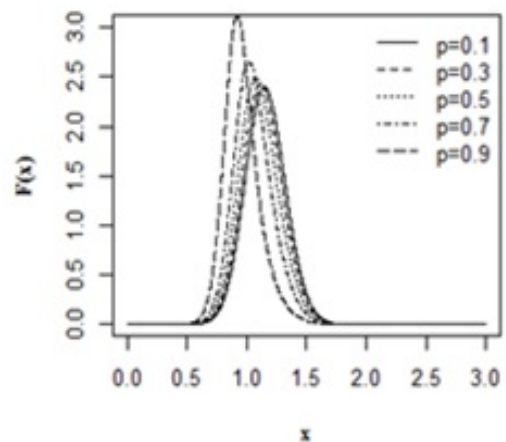

Figure 1: Pdf of the EWG distribution for selected values of the parameters.

Let $X=\min \left\{Y_{i}\right\}_{i=1}^{Z}$. Then the conditional pdf $X$ on $Z=z$ is given by

$$
\begin{aligned}
g(\mathbf{x} \mid z, \alpha, \beta) & =z[1-G(x \mid \alpha, \beta)]^{z-1} g(x \mid \alpha, \beta) \\
& =z \alpha \beta x^{\alpha-1} e^{-x^{\alpha}}\left(1-e^{-x^{\alpha}}\right)^{\beta-1}\left(1-\left(1-e^{-x^{\alpha}}\right)^{\beta}\right)^{z-1},
\end{aligned}
$$

where $g(x \mid \alpha, \beta)$ and $G(x \mid \alpha, \beta)$ are in (1.1) and (2.1), respectively. Therefore the marginal pdf of $\mathrm{X}$ is given by

$$
\begin{aligned}
f(\mathbf{x} \mid p, \alpha, \beta) & =\sum_{z=1}^{\infty} z \alpha \beta x^{\alpha-1} e^{-x^{\alpha}}\left(1-e^{-x^{\alpha}}\right)^{\beta-1}\left(1-\left(1-e^{-x^{\alpha}}\right)^{\beta}\right)^{z-1}(1-p) p^{z-1} \\
& =\frac{\alpha \beta(1-p) x^{\alpha-1} e^{-x^{\alpha}}\left(1-e^{-x^{\alpha}}\right)^{\beta-1}}{\left(1-p\left(1-\left(1-e^{-x^{\alpha}}\right)^{\beta}\right)\right)^{2}},
\end{aligned}
$$

which defines the exponentiated Weibull geometric (EWG) denoted by $\operatorname{EWG}(p, \alpha, \beta)$. It is clear that (2.4) is more flexible than the EW distribution. The exponentiated exponential geometric (EEG) distribution is a specal case of the EWG distribution for $\alpha=1$. When $p$ approaches zero, the EWG distribution leads to the EW distribution. Figure 1 shows the EWG density for various values of the 
parameter $(\alpha, \beta)$ when $p=0.1,0.3,0.5,0.7$ and 0.9 . For all values of parameters, the density tends to zero as $x \rightarrow \infty$.

If $|z|<1$ and $k>0$, we have the series representation

$$
(1-z)^{-k}=\sum_{j=0}^{\infty} \frac{\Gamma(k+j)}{\Gamma(k) j !} z^{j}
$$

Expanding $\left.\left\{1-p\left(1-\left(1-e^{-x^{\alpha}}\right)^{\beta}\right)\right)^{-2}\right\}$ as in $(2.5)$, we can write (2.4) as

$$
\begin{aligned}
f(\mathbf{x} \mid p, \alpha, \beta) & =\alpha \beta(1-p) x^{\alpha-1} e^{-x^{\alpha}}\left(1-e^{-x^{\alpha}}\right)^{\beta-1} \sum_{j=0}^{\infty}(j+1) p^{j}\left(1-\left(1-e^{x^{\alpha}}\right)^{\beta}\right)^{j} \\
& =\sum_{j=0}^{\infty} \sum_{k=0}^{\infty}(-1)^{k}\left(\begin{array}{c}
j+1 \\
j-k
\end{array}\right) p^{j} g(\mathbf{x} ; \alpha, \beta(k+1)),
\end{aligned}
$$

where $g(\mathbf{x} ; \alpha, \beta(k+1))$ is defined in (1.1). The pdf of the EWG distribution can be expressed as an infinite mixture of EW distributions.

Hence, some mathematical properties (cdf, moments, percentiles, moment generating function, factorial moments, etc.) of the EWG distribution can be obtained using (2.6) from the corresponding properties of the EW distribution.

\section{Properties of the EWG Distribution}

\subsection{The distribution and hazard functions and order statistics.}

Let $X$ be a random variable such that $X$ is distributed to EWG distribution with parameters $(p, \alpha, \beta)$,

$$
X \sim \operatorname{EWG}(p, \alpha, \beta)
$$

The cdf is given by

$$
F(\mathbf{x})=\frac{\left(1-e^{-x^{\alpha}}\right)^{\beta}}{1-p\left(1-\left(1-e^{-x^{\alpha}}\right)^{\beta}\right)}, \quad x>0 .
$$

The survivor and hazard functions are

$$
S(\mathbf{x})=\frac{(1-p)\left(1-\left(1-e^{-x^{\alpha}}\right)^{\beta}\right)}{1-p\left(1-\left(1-e^{-x^{\alpha}}\right)^{\beta}\right)}, \quad x>0
$$

and

$$
h(\mathbf{x})=\frac{\alpha \beta x^{\alpha-1} e^{-x^{\alpha}}\left(1-e^{-x^{\alpha}}\right)^{\beta-1}}{\left(1-\left(1-e^{-x^{\alpha}}\right)^{\beta}\right)\left(1-p\left(1-\left(1-e^{-x^{\alpha}}\right)^{\beta}\right)\right)}, \quad x>0
$$

respectively. We illustrates some of the possible shapes of the hazard function for selected values of the $(\alpha, \beta)$ when $p=0.1,0.3,0.5,0.7$ and 0.9 in Figure 2. These plots show that the hazard function of the EWG distribution is quite flexible. 
$(0.8,0.8)$

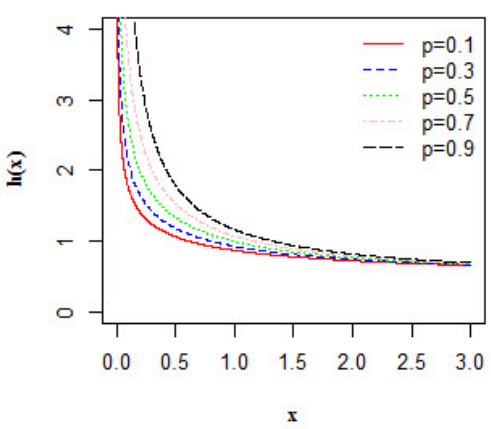

$(2,0.3)$

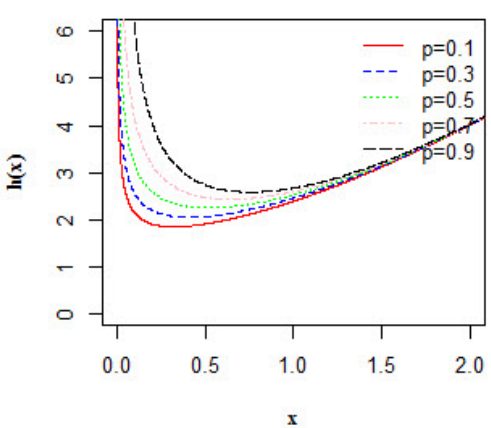

$(1.2,1.5)$

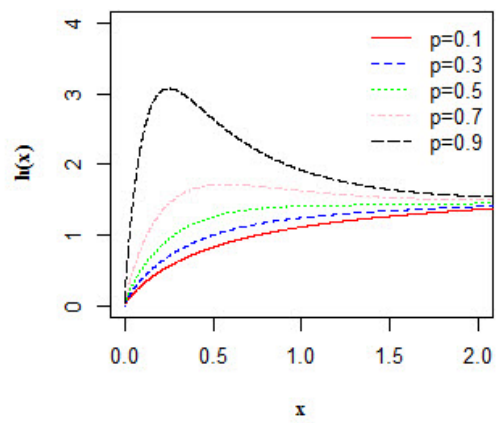

$(3,3)$

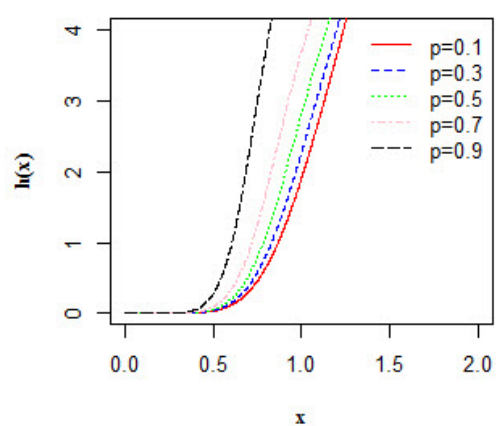

Figure 2: Hazard rate function of the EWG distribution for selected values of the parameters.

We now calculate the pdf of the order statistics. Let $g_{r: n}(x)$ be the pdf of the $r^{\text {th }}$ order statistic of the $\mathrm{EW}$ distirubion with parameters $\alpha, \beta$ given by

$$
g_{r: n}(\mathbf{x})=\frac{\alpha \beta x^{\alpha-1} e^{-x^{\alpha}}\left(1-e^{-x^{\alpha}}\right)^{\beta r-1}\left(1-\left(1-e^{-x^{\alpha}}\right)^{\beta}\right)^{n-r}}{B(r, n-r+1)} .
$$

where $B(a, b)=\int_{0}^{1} w^{a-1}(1-w)^{b-1} d w$ denotes the beta function.

Let $X_{1}, \ldots, X_{n}$ be random variables such that $X_{r} \sim \operatorname{EWG}(p, \alpha, \beta)$ independently for $r=1, \ldots, n$. The pdf of the $r^{\text {th }}$ order statistic, $X_{r: n}$ say, in given by, for $x>0$

$$
\begin{aligned}
f_{r: n}(\mathbf{x}) & =\frac{1}{B(r, n-r+1)} f(x)[F(x)]^{r-1}[1-F(x)]^{n-r} \\
& =\frac{\alpha \beta(1-p)^{n-r+1} x^{\alpha-1} e^{-x^{\alpha}}\left(1-e^{-x^{\alpha}}\right)^{\beta r-1}\left(1-\left(1-e^{-x^{\alpha}}\right)^{\beta}\right)^{n-r}}{B(r, n-r+1)\left(1-p\left(1-\left(1-e^{-x^{\alpha}}\right)^{\beta}\right)\right)^{n+1}},
\end{aligned}
$$

Equation (3.4) can be rewritten in terms of $g_{r: n}(x)$ in (3.3) as

$$
f_{r: n}(\mathbf{x})=(1-p)^{n-r+1}\left(1-p\left(1-\left(1-e^{-x^{\alpha}}\right)^{\beta}\right)\right)^{-(n+1)} g_{r: n}(x) .
$$


Further, we express the pdf of $X_{r: n}$ as a mixture distribution of EW order statistic densities. Using (2.5) and (3.5), we obtain

$$
\begin{aligned}
f_{r: n}(\mathbf{x})= & (1-p)^{n-r+1} \sum_{i=0}^{\infty} p^{i} \frac{(n+i) !}{n ! i !} \frac{n !}{(r-1) !(n-r) !} \frac{B(r, n+i-r+1)}{B(r, n+i-r+1)} \\
& * \alpha \beta x^{\alpha-1} e^{-x^{\alpha}}\left(1-e^{-x^{\alpha}}\right)^{\beta r-1}\left(1-\left(1-e^{-x^{\alpha}}\right)^{\beta}\right)^{b+i-r} \\
= & (1-p)^{n-r+1} \sum_{i=0}^{\infty} p^{i}\left(\begin{array}{c}
n+i-r \\
i
\end{array}\right) g_{r: n+i}(x),
\end{aligned}
$$

where $g_{r: n+i}(x)$ is defined in (3.3). Hence, using (3.6), some mathematical properties for the order statistics of the EWG distribution can be immediately obtained from the corresponding properties of the EW order statistics.

\subsection{Quantiles and moments}

The quantile $u\left(x_{u}\right)$ of the EWG distribution follows from (2.7) as

$$
x_{u}=\left(-\log \left(1-\left(\frac{u-p u}{1-p u}\right)^{\frac{1}{\beta}}\right)\right)^{\frac{1}{\alpha}} .
$$

In particular, the median is simply calculated as $x_{0.5}=\left(-\log \left(1-((1-p) /(2-p))^{1 / \beta}\right)\right)^{1 / \alpha}$. Suppose that $Y \sim \mathrm{EW}(\alpha, \beta)$. Then the $r^{\text {th }}$ moment of $Y$ is given by

$$
\begin{aligned}
E\left(Y^{r}\right) & =\int_{0}^{\infty} y^{r} \alpha \beta y^{\alpha-1} e^{-y^{\alpha}}\left(1-e^{-y^{\alpha}}\right)^{\beta-1} d y \\
& =\beta \sum_{j=0}^{\infty}\left(\begin{array}{c}
\beta-1 \\
j
\end{array}\right)(-1)^{j} \Gamma\left(\frac{r}{\alpha}+1\right)(j+1)^{-\left(\frac{r}{\alpha}+1\right)} .
\end{aligned}
$$

Suppose that $X \sim \operatorname{EWG}(p, \alpha, \beta)$. Then the $r^{\text {th }}$ moment of $X$ is given by

$$
\begin{aligned}
E\left(X^{r}\right)= & \sum_{j=0}^{\infty} \sum_{k=0}^{\infty}(-1)^{k}(1-p) p^{j}\left(\begin{array}{l}
j+1 \\
j-k
\end{array}\right) \int_{0}^{\infty} x^{r} \alpha \beta(k+1) x^{\alpha-1} e^{-x^{\alpha}}\left(1-e^{-x^{\alpha}}\right)^{\beta(k+1)-1} d x \\
= & \sum_{j=0}^{\infty} \sum_{k=0}^{\infty}(-1)^{k}(1-p) p^{j}\left(\begin{array}{c}
j+1 \\
j-k
\end{array}\right) \int_{0}^{\infty} x^{r} f_{E W}(\mathbf{x} ; \alpha, \beta(k+1)) d x \\
= & \sum_{i=0}^{\infty} \sum_{j=0}^{\infty} \sum_{k=0}^{\infty}(-1)^{i+k}(1-p) p^{j} \beta(k+1) \\
& *(i+1)^{-\left(\frac{r}{\alpha}+1\right)}\left(\begin{array}{c}
j+1 \\
j-k
\end{array}\right)\left(\begin{array}{c}
\beta(k+1)-1 \\
i
\end{array}\right) \Gamma\left(\frac{r}{\alpha}+1\right) .
\end{aligned}
$$


Using Equation (3.9), the moment generating function of the EWG distribution is given by

$$
\begin{aligned}
M_{X}(t)= & E\left[\sum_{r=0}^{\infty} \frac{t^{r}}{r !} X^{r}\right] \\
= & \sum_{i=0}^{\infty} \sum_{j=0}^{\infty} \sum_{k=0}^{\infty} \sum_{r=0}^{\infty} \frac{t^{r}}{r !}(-1)^{i+k}(1-p) p^{j} \beta(k+1) \\
& *(i+1)^{-\left(\frac{r}{\alpha}+1\right)}\left(\begin{array}{c}
j+1 \\
j-k
\end{array}\right)\left(\begin{array}{c}
\beta(k+1)-1 \\
i
\end{array}\right) \Gamma\left(\frac{r}{\alpha}+1\right) .
\end{aligned}
$$

The $k^{\text {th }}$ moment of the $r^{\text {th }}$ order statistics $Y_{r: n}$ had on $Y_{1}, \ldots, Y_{n}$ from $\operatorname{EW}(\alpha, \beta)$ is given by

$$
E\left(Y_{r: n}^{k}\right)=\frac{\alpha \beta}{B(r, n-r+1)} \sum_{j=0}^{n-i} \sum_{s=0}^{\infty}(-1)^{j+s} \Gamma\left(\frac{k}{\alpha}+1\right)(k+1)^{-\left(\frac{k}{\alpha}+1\right)}\left(\begin{array}{c}
n-r \\
j
\end{array}\right)\left(\begin{array}{c}
\beta(r+j)-1 \\
s
\end{array}\right) .
$$

The $k^{\text {th }}$ moment of the $r^{\text {th }}$ order statistics $X_{r: n}$ had on $X_{1}, \ldots, X_{n}$ from $\operatorname{EWG}(p, \alpha, \beta)$ is given by

$$
\begin{aligned}
E\left(X_{r: n}^{k}\right) & =\int_{0}^{\infty} x^{k}(1-p)^{n-r+1} \sum_{i=0}^{\infty} p^{i}\left(\begin{array}{c}
n+i-r \\
i
\end{array}\right) g_{r: n+i}(x) d x \\
& =(1-p)^{n-r+1} \sum_{i=0}^{\infty} p^{i}\left(\begin{array}{c}
n+i-r \\
i
\end{array}\right) \int_{0}^{\infty} x^{k} g_{r: n+i}(x) d x .
\end{aligned}
$$

We can express the $r^{\text {th }}$ moment of $X_{r: n}$ as mixture of EW order statistic densities as follows;

$$
E\left(X_{r: n}^{k}\right)=(1-p)^{n-r+1} \sum_{i=0}^{\infty} p^{i}\left(\begin{array}{c}
n+i-r \\
i
\end{array}\right) E_{E W}\left[X_{r: n+i}^{k}\right]
$$

\subsection{Renyi and Shannon entropies}

Entropy has widely been used in various situations in engineering. The entropy of a random variable $X$ is a measure of variation of the uncertainty. Renyi entropy is defined by $I_{R}(r)=1 /(1-r) \log \left(\int_{R} f^{r}(x)\right.$ $d x$ ), for $r>0$ and $r \neq 1$. For the EWG distribution, the power series expansion gives

$$
\begin{aligned}
\int_{0}^{\infty} f^{r}(x) d x= & {[\alpha \beta(1-p)]^{r} \sum_{j=0}^{\infty} \frac{\Gamma(2 r+j)}{\Gamma(2 r) j !} p^{j} \int_{0}^{\infty} x^{r(\alpha-1)}\left(e^{-x^{\alpha}}\right)^{r}\left(1-e^{-x^{\alpha}}\right)^{r(\beta-1)}\left(1-\left(1-e^{-x^{\alpha}}\right)^{\beta}\right)^{j} d x } \\
= & {[\alpha \beta(1-p)]^{r} \sum_{j=0}^{\infty} \sum_{k=0}^{\infty} \sum_{l=0}^{\infty} p^{j}(-1)^{(k+l)} \frac{\Gamma(2 r+j)}{\Gamma(2 r) j !} \frac{\Gamma(j+1)}{\Gamma(j+1-k) k !} } \\
& * \frac{\Gamma(\beta(r+k)-r+1)}{\Gamma(\beta(r+k)-r-l) l !} \Gamma\left(\frac{r \alpha-r+1}{\alpha}\right)(r+l)^{-\frac{r \alpha-r+1}{\alpha}} .
\end{aligned}
$$

Substituting from (3.14), the Renyi entropy is given by

$$
\begin{aligned}
I_{R}(r)= & \frac{1}{1-r} \log \left\{[\alpha \beta(1-p)]^{r} \sum_{j=0}^{\infty} \sum_{k=0}^{\infty} \sum_{l=0}^{\infty} p^{j}(-1)^{(k+l)} \frac{\Gamma(2 r+j)}{\Gamma(2 r) j !} \frac{\Gamma(j+1)}{\Gamma(j+1-k) k !}\right. \\
& \left.* \frac{\Gamma(\beta(r+k)-r+1)}{\Gamma(\beta(r+k)-r-l) l !} \Gamma\left(\frac{r \alpha-r+1}{\alpha}\right)(r+l)^{-\frac{r \alpha-r+1}{\alpha}}\right\} .
\end{aligned}
$$


The Shannon entropy which is defined by $E[-\log (f(X))]$, is derived from $\lim _{r \rightarrow 1} I_{R}(r)$.

\section{Estimation}

\subsection{Maximum Likelihood Estimator (MLE)}

Let $x=\left(x_{1}, \ldots, x_{n}\right)$ be a random sample from the EWG distribution in (2.4) with unknown parameter vector $\theta=(p, \alpha, \beta)^{\prime}$. Then the $\log$ likelihood function $\ell=\ell(\theta ; x)$ for $\theta$ is

$$
\begin{aligned}
\ell= & n \log \alpha+n \log \beta+n \log (1-p)+(\alpha-1) \sum_{i=1}^{n} \log \left(x_{i}\right) \\
& -\sum_{i=1}^{n} x_{i}^{\alpha}+(\beta-1) \sum_{i=1}^{n} \log \left(1-e^{-x_{i}^{\alpha}}\right)-2 \sum_{i=1}^{n} \log \left(1-p\left(1-e^{-x_{i}^{\alpha}}\right)^{\beta}\right) .
\end{aligned}
$$

The score function $U(\theta)=(\partial \ell / \partial p, \partial \ell / \partial \alpha, \partial \ell / \partial \beta)^{T}$ is based on a random sample of the EWG distribution in (2.4) has components as follows;

$$
\begin{aligned}
\frac{\partial \ell}{\partial \alpha}= & \frac{n}{\alpha}+\sum_{i=1}^{n} \log \left(x_{i}\right)-\sum_{i=1}^{n} x_{i}^{\alpha} \log \left(x_{i}\right)+\sum_{i=1}^{n} \frac{(\beta-1) x_{i}^{\alpha} \log \left(x_{i}\right) e^{-x_{i}^{\alpha}}}{1-e^{-x_{i}^{\alpha}}} \\
& -2 \sum_{i=1}^{n} \frac{p \beta x_{i}^{\alpha} \log \left(x_{i}\right) e^{-x_{i}^{\alpha}}\left(1-e^{\left.-x_{i}^{\alpha}\right)^{\beta-1}}\right.}{1-p\left(1-\left(1-e^{-x_{i}^{\alpha}}\right)^{\beta}\right)}, \\
\frac{\partial \ell}{\partial \beta}= & \frac{n}{\beta}+\sum_{i=1}^{n} \log \left(1-e^{-x_{i}^{\alpha}}\right)+2 \sum_{i=1}^{n} \frac{p\left(1-e^{-x_{i}^{\alpha}}\right)^{\beta} \log \left(1-e^{-x_{i}^{\alpha}}\right)}{1-p\left(1-\left(1-e^{-x_{i}^{\alpha}}\right)^{\beta}\right)} \\
\frac{\partial \ell}{\partial p}= & -\frac{n}{1-p}+2 \sum_{i=1}^{n} \frac{1-\left(1-e^{-x_{i}^{\alpha}}\right)^{\beta}}{1-p\left(1-\left(1-e^{-x_{i}^{\alpha}}\right)^{\beta}\right)} .
\end{aligned}
$$

The maximum likelihood estimate (MLE) $\hat{\theta}$ of $\theta$ is calculated numerically from the nonlinear equations $\mathrm{U}(\theta)=0$. Here, we use the EM algorithm (Dempster et al., 1977; McLachlan and Krishnan, 1997) to obtain $\hat{\theta}$. First of all, we can obtain a complete likelihood function of as follows;

$$
\begin{aligned}
L_{c}(\theta) & =\prod_{i=1}^{n} g\left(x_{i} \mid z_{i}, \theta\right) p\left(z_{i} \mid \theta\right) \\
& =[\alpha \beta(1-p)]^{n} \prod_{i=1}^{n}\left\{t z_{i} x_{i}^{\alpha-1} e^{-x_{i}^{\alpha}}\left(1-e^{-x_{i}^{\alpha}}\right)^{\beta-1}\left[p\left(1-\left(1-e^{-x_{i}^{\alpha}}\right)^{\beta}\right)\right]^{z_{i}-1}\right\} .
\end{aligned}
$$

The complete log-likelihood function of $\theta$ is

$$
\begin{aligned}
\log L_{c}(\theta)= & n \log \alpha+n \log \beta+n \log (1-p)+\sum_{i=1}^{n} \log z_{i}+(\alpha-1) \sum_{i=1}^{n} \log x_{i}-\sum_{i=1}^{n} x_{i}^{\alpha} \\
& +(\beta-1) \sum_{i=1}^{n} \log \left(1-e^{-x_{i}^{\alpha}}\right)+\log p \sum_{i=1}^{n}\left(z_{i}-1\right)+\sum_{i=1}^{n} \log \left(1-\left(1-e^{-x_{i}^{\alpha}}\right)^{\beta}\right)\left(z_{i}-1\right) .
\end{aligned}
$$


Therefore, the E-step is $Q\left(\theta: \theta_{(k)}\right)=E_{\theta_{(k)}}\left[\log L_{c}(\theta) \mid X, \theta\right]$ where $\theta_{(k)}$ denotes the expectation with respect to the conditional density of $Z$ given $X$ and $\theta$ evaluated at $\left(\theta_{(k)}, \theta\right)$ that is, the $k^{\text {th }}$ current estimate of $\theta$. The conditional density of $Z$ given $X$ and $\theta$ is

$$
P\left(z_{i} \mid x_{i}, \theta\right)=z_{i}\left[p\left(1-\left(1-e^{-x_{i}^{\alpha}}\right)^{\beta}\right)\right]^{z_{i}-1}\left[1-p\left(1-\left(1-e^{-x_{i}^{\alpha}}\right)^{\beta}\right)\right]^{2}
$$

and the conditional expectation of $Z$ given $X$ and $\theta$ is given by

$$
\begin{aligned}
E\left[z_{i} \mid x_{i}, \theta\right] & \left.=\sum_{z=1}^{\infty} z_{i}^{2}\left[p\left(1-\left(1-e^{-x^{\alpha}}\right)^{\beta}\right)\right]^{z_{i}-1}\left[1-p\left(1-\left(1-e^{-x^{\alpha}}\right)\right]^{\beta}\right)\right]^{2} \\
& =\frac{1+p\left(1-\left(1-e^{-x^{\alpha}}\right)^{\beta}\right)}{1-p\left(1-\left(1-e^{-x^{\alpha}}\right)^{\beta}\right)} \\
& =W_{i}(p, \alpha, \beta) . \quad \text { (say) }
\end{aligned}
$$

Now, the E-step is given as follows;

$$
\begin{aligned}
Q\left(\theta: \theta_{(k)}\right)= & E_{\theta_{(k)}}\left[\log L_{c}(\theta) \mid X, \theta\right] \\
= & n \log \alpha+n \log \beta+n \log (1-p)+\sum_{i=1}^{n} E_{\theta_{(k)}}\left[\log z_{i} \mid x_{i}, \theta\right]+(\alpha-1) \sum_{i=1}^{n} \log x_{i} \\
& -\sum_{i=1}^{n} x_{i}^{\alpha}+(\beta-1) \sum_{i=1}^{n} \log \left(1-e^{-x_{i}^{\alpha}}\right)+\log p \sum_{i=1}^{n}\left(W_{i}(p, \alpha, \beta)-1\right) \\
& +\sum_{i=1}^{n} \log \left(1-\left(1-e^{-x_{i}^{\alpha}}\right)^{\beta}\right)\left(W_{i}(p, \alpha, \beta)-1\right) \\
= & Q^{*}\left(\theta: \theta_{(k)}\right)+\sum_{i=1}^{n} E_{\theta_{(k)}}\left[\log z_{i} \mid x_{i}, \theta\right] .
\end{aligned}
$$

Since the term of $E_{\theta_{(k)}}[\log z \mid X, \theta]$ is not needed for maximizing $Q\left(\theta ; \theta_{(k)}\right)=E_{\theta_{(k)}}\left[\log L_{c}(\theta) \mid X, \theta\right]$, maximizing $Q\left(\theta: \theta_{(k)}\right)$ is equivalent to maximaing $Q^{*}\left(\theta: \theta_{(k)}\right)$ where

$$
\begin{aligned}
Q^{*}\left(\theta: \theta_{(k)}\right)= & n \log \alpha+n \log \beta+n \log (1-p)+(\alpha-1) \sum_{i=1}^{n} \log x_{i} \\
& -\sum_{i=1}^{n} x_{i}^{\alpha}+(\beta-1) \sum_{i=1}^{n} \log \left(1-e^{-x_{i}^{\alpha}}\right)+\log p \sum_{i=1}^{n}\left(W_{i}^{(k)}-1\right) \\
& +\sum_{i=1}^{n} \log \left(1-\left(1-e^{-x_{i}^{\alpha}}\right)^{\beta}\right)\left(W_{i}^{(k)}-1\right) .
\end{aligned}
$$

Next, let $\theta_{(k+1)}=\operatorname{argmax} Q^{*}\left(\theta ; \theta_{(k)}\right)$ in (4.6) over $\theta$. Then $\theta_{(k+1)}=\left(p^{(k+1)}, \alpha^{(k+1)}, \beta^{(k+1)}\right)$ is obtained as 
follows;

$$
\begin{aligned}
\frac{\partial Q^{*}\left(\theta: \theta_{(k)}\right)}{\partial p}= & -\frac{1}{1-p}+\frac{1}{p} \sum_{i=1}^{n} W_{i}^{(k)}, \quad p_{(k+1)}=1-\frac{n}{\sum_{i=1}^{n} W_{i}^{(k)}}, \\
\frac{\partial Q^{*}\left(\theta: \theta_{(k)}\right)}{\partial \alpha}= & \frac{m}{\alpha^{(k+1)}}+\sum_{i=1}^{n} \log x_{i}-\sum_{i=1}^{n} x_{i}^{\alpha^{(k+1)}} \log x_{i}+\left(\beta^{(k+1)}-1\right) \sum_{i=1}^{n} \frac{x_{i}^{\alpha^{(k+1)}} \log x_{i} e^{-x_{i}^{\alpha^{(k+1)}}}}{1-e^{-x_{i}^{\left(k^{(k+1)}\right.}}} \\
& -\sum_{i=1}^{n} \frac{\beta^{(k+1)} x_{i}^{\alpha^{(k+1)}} \log x_{i} e^{-x_{i}^{\alpha^{(k+1)}}}\left(1-e^{-x_{i}^{\alpha^{(k+1)}}}\right)^{\beta^{(k+1)}-1}}{1-\left(1-e^{-x_{i}^{\alpha^{(k+1)}}}\right)^{\beta^{(k+1)}}}\left(W_{i}^{(k)}-1\right)
\end{aligned}
$$

and

$$
\begin{aligned}
\frac{\partial Q^{*}\left(\theta: \theta_{(k)}\right)}{\partial \beta}= & \frac{n}{\beta}+\sum_{i=1}^{n} \log \left(1-e^{-x_{i}^{\alpha^{(k+1)}}}\right) \\
& -\sum_{i=1}^{n} \frac{\left(1-e^{-x_{i}^{\alpha^{(k+1)}}}\right)^{\beta^{(k+1)}} \log \left(1-e^{-x_{i}{ }^{(k+1)}}\right)}{1-\left(1-e^{-x_{i}^{\alpha^{(k+1)}}}\right)^{\beta^{(k+1)}}}\left(W_{i}^{(k)}-1\right),
\end{aligned}
$$

where $W_{i}^{(k)}=W_{i}\left(p^{(k)}, \alpha^{(k)}, \beta^{(k)}\right)$ in (4.5).

\subsection{Bayesian estimation}

Assume that $p, \alpha$ and $\beta$ are independent. The prior $\pi_{0}(p)$ for $p$ is assumed to be beta distribution with parameters $a_{0}$ and $b_{0}$ and each prior for $\alpha, \beta$ is gamma distribution as follows;

$$
\begin{aligned}
& \pi_{0}(p)=\frac{1}{B\left(a_{0}, b_{0}\right)} p^{a_{0}-1}(1-p)^{b_{0}-1}, \\
& \pi_{1}(\alpha)=\frac{b_{1}^{a_{1}}}{\Gamma\left(a_{1}\right)} \alpha^{a_{1}-1} e^{-b_{1} \alpha}
\end{aligned}
$$

and

$$
\pi_{2}(\beta)=\frac{b_{2}^{a_{2}}}{\Gamma\left(a_{2}\right)} \beta^{a_{2}-1} e^{-b_{2} \beta},
$$

where $a_{i}$ and $b_{i}$ are known for $i=0,1,2$.

The joint posterior density for $p, \alpha, \beta$ given the observed data $x_{1}, \ldots, x_{n}$ from EWG in (2.4) is obtained as follows;

$$
\begin{aligned}
\pi\left(p, \alpha, \beta \mid x_{1}, \ldots, x_{n}\right) \propto & \prod_{i=1}^{n} \frac{\alpha \beta(1-p) x_{i}^{\alpha-1} e^{-x_{i}^{\alpha}}\left(1-e^{-x_{i}^{\alpha}}\right)^{\beta-1}}{\left(1-p\left(1-\left(1-e^{-x_{i}^{\alpha}}\right)^{\beta}\right)\right)^{2}} \\
& * p^{a_{0}-1}(1-p)^{b_{0}-1} \alpha^{a_{1}-1} e^{b_{1} \alpha} \beta^{a_{1}-1} e^{b_{1} \beta} .
\end{aligned}
$$


Table 1: MLEs and Bayesian estimates for the plasma concentrations of indomethacin data

\begin{tabular}{cccc}
\hline \hline EWG model & $p$ & $\alpha$ & $\beta$ \\
\hline \multirow{2}{*}{ MLEs } & 0.7672 & 1.0210 & 1.3125 \\
& $(0.0345)$ & $(0.1424)$ & $(0.1653)$ \\
\hline \multirow{2}{*}{ Bayesian estimates } & 0.7753 & 1.2536 & 1.2960 \\
& $(0.0319)$ & $(0.1397)$ & $(0.2016)$ \\
\hline \hline
\end{tabular}

Therefore, Gibbs sampling (Gelfand and Smith, 1990) the full conditional distributions (FCD) are obtained from (4.8) as follows;

$$
\begin{aligned}
& \pi\left(p \mid \alpha, \beta, x_{1}, \ldots, x_{n}\right) \propto p^{a_{0}-1}(1-p)^{b_{0}-1} \prod_{i=1}^{n} \frac{p}{\left(1-p\left(1-\left(1-e^{-x_{i}^{\alpha}}\right)^{\beta}\right)\right)^{2}}, \\
& \pi\left(\alpha \mid p, \beta, x_{1}, \ldots, x_{n}\right) \propto \alpha^{a_{1}-1} e^{b_{1} \alpha} \prod_{i=1}^{n} \frac{\alpha x_{i}^{\alpha} e^{-x_{i}^{\alpha}}\left(1-e^{-x_{i}^{\alpha}}\right)^{\beta-1}}{\left(1-p\left(1-\left(1-e^{-x_{i}^{\alpha}}\right)^{\beta}\right)\right)^{2}},
\end{aligned}
$$

and

$$
\pi\left(\beta \mid p, \alpha, x_{1}, \ldots, x_{n}\right) \propto \beta^{a_{2}-1} e^{b_{2} \beta} \prod_{i=1}^{n} \frac{\beta\left(1-e^{-x_{i}^{\alpha}}\right)^{\beta}}{\left(1-p\left(1-\left(1-e^{-x_{i}^{\alpha}}\right)^{\beta}\right)\right)^{2}} .
$$

For generating random samples of $p, \alpha$ and $\beta$ in Gibbs sampling, we use the Metropolis-Hastings algorithm (Metropolis et al., 1953; Hastings, 1970) because of having intractable full conditional distributions.

\section{Application to Real Life Data}

In this section, we fit the EWG models to two real data sets. The first data set is about the plasma concentrations of indomethacin following intravenous injection. We used the pooled data with 66 observation in Kwan et al. (1976). The second data set consists of 128 observations on phosphorus concentration in the leaves which is studied by Fonseca and Franca (2007). For the Bayesian inference of these two data set, flat priors are used in the absense of any strong prior information,. That is, the prior $\pi_{0}(p)$ for $\mathrm{p}$ is assumed to be beta distribution with parameters $a_{0}=1$ and $b_{0}=1$. Each prior for $\alpha, \beta$ is gamma distribution with parameters $a_{1}=a_{2}=1$ and $b_{1}=b_{2}=0$. Then, the joint posterior density is proportional to the likelihood function. In order to obtain the Bayes estimates of $p, \alpha$ and $\beta$, we apply the Metropolis-Hasting algorithms into the FCDs (4.9) within Gibbs sampler. Here, the each prior distributions are used as the proposed distributions in Metropolis-Hastings algorithm steps. Then, we run the Gibbs sampler to generate a Markov chain with 50,000 samples. Discarding the first 10,000 values as burn-in and taking every fifth variates as iid samples.

Example 1. For the plasma concentrations of indomethacin data set, we obtain the Bayeses estimates and MLEs of parameters from EWG model in Table 1.

Next, we fitted the EWG, EW, generalized exponential (GE) and Weibull (W) distribution to these data by the method of maximum likelihood. The MLEs of the parameters and the Akaike information 


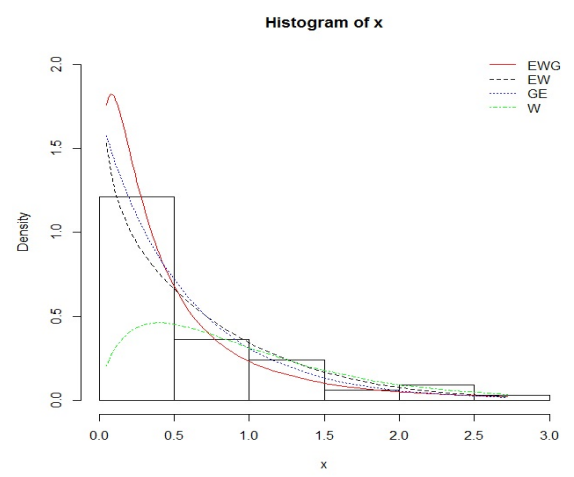

Figure 3: Fitted EWG, EW, GE and Weibull dinsities for phosphorus concentration in leaves data.

Table 2: MLEs of the model parameters for the plasma concentrations of indomethacin data, the AIC and BIC measures.

\begin{tabular}{cccccc}
\hline \hline Model & $p$ & $\alpha$ & $\beta$ & AIC & BIC \\
\hline EWG & 0.7672 & 1.0210 & 1.3125 & 64.9393 & 71.5082 \\
EW & 0 & 1.3650 & 0.5596 & 69.9762 & 74.3555 \\
GE & 0 & 1.6714 & 0.9836 & 66.7482 & 71.1275 \\
W & 0 & 1 & 0.9366 & 79.7124 & 81.9020 \\
\hline \hline
\end{tabular}

Table 3: MLEs and Bayesian estimates for phosphorus concentration in leaves data

\begin{tabular}{cccc}
\hline \hline EWG model & $p$ & $\alpha$ & $\beta$ \\
\hline \multirow{2}{*}{ MLEs } & 0.9999 & 8.7544 & 0.4793 \\
& $(0.2196)$ & $(0.3951)$ & $(0.3862)$ \\
\hline \multirow{2}{*}{ Bayesian estimates } & 0.9982 & 8.3961 & 0.6153 \\
& $(0.1975)$ & $(0.3862)$ & $(0.3810)$ \\
\hline \hline
\end{tabular}

criterion (AIC) and Bayesian information criterion (BIC) for the fitted models are displayed in Table 2.

Our first comparison is based on the likelihood ratio test (LRT) of $H_{0}: p=0$ (EW model) against $H_{1}: p \neq 0$ (EWG model).

The LRT can be used, based on the fact that a $\log$-likelihood $(\log L)$ ratio statistic is asymptotically chi-square distributed with one degree of freedom. Since the LR statistic for testing the hypotheses $H_{0}$ vs. $H_{1}$ is 7.0369 , we reject the null hypotheses $H_{0}: p=0$ (EW model). That is, the EWG distribution provides a significantly better goodness of fit than the EW distribution. The plots of the fitted EWG, EW, GE and $\mathrm{W}$ densities are shown in Figure 3. It indicates that the proposed distribution provides better goodness of fit than the other sub-models.

Example 2. For the second data set, we obtain the Bayeses estimates and MLEs of parameters from EWG model in Table 3.

Next, we fitted the EWG, EW, GE and W distribution to these data by the method of maximum likelihood. The MLEs of the parameters and the AIC and BIC for the fitted models are displayed in Table 4.

The LR statistic for testing the hypotheses $H_{0}$ vs $H_{1}$ is 301.0515 . So, we reject the null hypotheses in this case in favour of the EWG distribution. The plots of the fitted EWG, EW, GE and W densities 
Table 4: MLEs of the model parameters for the phosphorus concentration in leaves data, the AIC and BIC measures.

\begin{tabular}{cccccc}
\hline \hline Model & $\mathrm{p}$ & $\alpha$ & $\beta$ & AIC & BIC \\
\hline EWG & 0.9999 & 8.7544 & 0.4793 & -378.519 & -369.963 \\
EW & 0 & 9.6050 & 0.0510 & -79.468 & -73.761 \\
GE & 0 & 21.1327 & 10.6058 & -388.043 & -382.338 \\
W & 0 & 1 & 0.6596 & -0.0007 & 2.8513 \\
\hline \hline
\end{tabular}

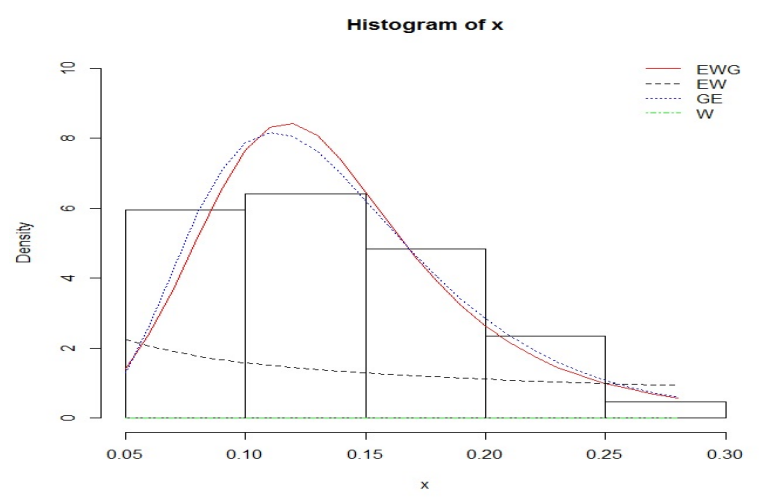

Figure 4: Fitted EWG, EW, GE and Weibull dinsities for phosphorus concentration in leaves data.

are shown in Figure 4. It indicates that the proposed distribution provides better goodness of fit than the other sub-models.

\section{Summary and Discussion}

We introduce a new model called the exponentiatedWeibull-geometric (EWG) distribution which generalizes two-parameter exponentiated Weibull (EW) distribution, whose failure rate function can be increasing, decreasing and bathtub-shaped. This proposed distribution is obtained by compounding the exponentiated Weibull with geometric distribution. Its density function can be expressed as a mixture of exponentiatedWeibull densities. Also, we derive its cumulative distribution function (CDF), hazard function and the density of the order statistics and calculate expressions for its moments and the mo- ments of the order statistics. We give expressions for the Renyi and Shannon entropies. The maximum likelihood estimation and Bayesian estimation are obtained by using EM-algorithm (Dempster $e t$ al., 1977; McLachlan and Krishnan, 1997) and by using Gibbs sampler with Metropolis-Hastings algorithm respectively. Fianlly, we present the use of LR statistics to compare the fit of the EWG model with EW model. Also, we fitted EWG model to real data sets to show the exibility and potentially of the new model using AIC and BIC. Further works includes compounding the three-parameter exponential Weibull distribution with the geometric distribution and obtaining its properties and estimations on progressively type II censoring. Especially, Lindley method, Tierney-Kadane approximation and importance sampling method for computing Bayes estimation will be investigated.

\section{References}

Alexander, C., Cordeiro, G. M., Ortega, E. M. M. and Sarabia, J. M. (2012). Generalized betagenerated distributions, Computational Statistics and Data Analysis, 56, 1880-1897. 
Azzalini, A. and Capitanio, A. (2003). Distribution generated by perturbation of symmetry with emphasis on a multivariate skew $t$-distribution, Journal of the Royal Statistical Society, Series B, 65, 367-389.

Barreto-Souza, W., Lemos de Morais, A. and Cordeiro, G. M. (2011). The Weibull geometric distribution, Journal of Statistical Computation and Simulation, 81, 645-657.

Bidram, H., Behboodian, J. and Towhidi, M. (2011) The beta Weibull-geometric distribution, Jornal of Statistical Computation and Simulation, 83, 52-67.

Cordeiro, G. M., Silva, G. O. and Ortega, M. M. (2011) The beta-Weibull geometric distribution, Statistics, DOI:10.1080/02331888.2011.577897

Dempster, A. P., Laird, N. M. and Rubin, D. B. (1977). Maximum likelihood from incomplete data via the EM algorithm, Journal of the Royal Statistical Society, Series B (Methodological), 39, $1-38$.

Eugene, N., Lee, C. and Famoye, F. (2002). Beta-normal distribution and its applications, Communications in Statistics - Theory and Methods, 31, 497-512.

Fonseca, M. B. and Franca, M. G. C. (2007). A in uencia da fertilidade do solo e caracterizacao da xacao biologica de N2 para o crescimento de Dimorphandra wilsonii rizz, Master's thesis, Universidade Federal de Minas Gerais.

Gelfand, E. and Smith, F. M. (1990). Sampling-Based approaches to calculating marginal densities, Journal of the American Statistical Association, 85, 398-409.

Hansen, B. E. (1994). Autoregressive conditional density estimation, International Economic Review, 35, 705-730.

Hastings, W. K. (1970). Monte Carlo sampling methods using Markov chains and their applications, Biometrika, 57, 97-109.

Jung, J. and Chung, Y. (2013). Bayesian prediction of Exponential Weibull distribution based on progressive type II cednsoring, Communications for Statistical Applications and Methods, 20, $427-438$.

Kim, C., Jung, J. and Chung, Y. (2011). Bayesian estimation for the exponentiated Weibull model under Type II progressive censoring, Statistical Papers, 52, 53-70.

Kwan, K. C., Breault, G. O., Umbenhauer, E. R., McMahon, F. G. and Duggan, D. F. (1976). Kinetics of Indomethacin absorption, elimination, and enterohepatic circulation in man, Journal of Pharmacokinetics and Biopharmaceutics, 4, 255-280.

Mahmoudi, E. and Shiran, M. (2012). Exponentiated Weibull-Geometric Distribution and its Applications, arXiv:1206.4008vl [stat.ME]

McLachlan, G. J. and Krishnan, T. (1997). The EM Algorithm and Extension, Wiley, New York.

Metropolis, N., Rosenbluth, A. W., Rosenbluth, M. N., Teller, A. H. and Teller, E. (1953). Equations of state calculations by fast computing machines, Journal of Chemical Physics, 21, 1087-1092.

Mudholkar, G. S., Srivastava, D. K. and Freimer, M. (1995). The exponentiated Weibull family; a reanalysis of the bus motor failure data, Technometrics, 37, 436-445.

Nadarajah, S., Cordeiro, G. M. and Ortega, E. M. M. (2013). The exponentiated Weibull distribution: A survey, Statistical Papers, 54, 839-877.

Nassar, M. M. and Eissa, F. H. (2003). On the exponentiated Weibull distribution, Communications in Statistics - Theory and Methods, 32, 1317-1336. doi:10.1081/STA-120021561.

Nassar, M. M. and Eissa, F. H. (2004). Bayesian estimation for the exponentiated Weibull model, Communications in Statistics - Theory and Methods, 33, 2343-2362. 